\title{
Structural and electronic properties of $\mathrm{MoS}_{2}, \mathrm{WS}_{2}$, and $\mathrm{WS}_{2} / \mathrm{MoS}_{2}$ heterostructures encapsulated with hexagonal boron nitride monolayers
}

\author{
C. Yelgel, ${ }^{1}{ }^{1, a)}$ Ö. C. Yelgel, ${ }^{1}$ and O. Gülseren ${ }^{2}$ \\ ${ }^{1}$ Faculty of Engineering, Department of Materials Science and Nanotechnology Engineering, \\ Recep Tayyip Erdogan University, 53100 Rize, Turkey \\ ${ }^{2}$ Department of Physics, Bilkent University, 06800 Ankara, Turkey
}

(Received 29 May 2017; accepted 31 July 2017; published online 10 August 2017)

\begin{abstract}
In this study, we investigate the structural and electronic properties of $\mathrm{MoS}_{2}, \mathrm{WS}_{2}$, and $\mathrm{WS}_{2} / \mathrm{MoS}_{2}$ structures encapsulated within hexagonal boron nitride (h-BN) monolayers with first-principles calculations based on density functional theory by using the recently developed non-local van der Waals density functional (rvv10). We find that the heterostructures are thermodynamically stable with the interlayer distance ranging from $3.425 \AA$ to $3.625 \AA$ implying van der Waals type interaction between the layers. Except for the $\mathrm{WS}_{2} / \mathrm{h}-\mathrm{BN}$ heterostructure which exhibits direct band gap character with the value of $1.920 \mathrm{eV}$ at the $\mathrm{K}$ point, all proposed heterostructures show indirect band gap behavior from the valence band maximum at the $\Gamma$ point to the conduction band minimum at the $\mathrm{K}$ point with values varying from $0.907 \mathrm{eV}$ to $1.710 \mathrm{eV}$. More importantly, it is found that h-BN is an excellent candidate for the protection of intrinsic properties of $\mathrm{MoS}_{2}, \mathrm{WS}_{2}$, and $\mathrm{WS}_{2} / \mathrm{MoS}_{2}$ structures. Published by AIP Publishing. [http://dx.doi.org/10.1063/1.4998522]
\end{abstract}

\section{INTRODUCTION}

The successful experimental realization of monolayer graphene leads to a new research field with vast amount of interest in two dimensional (2D) structures because of their fascinating properties. The extent of research on $2 \mathrm{D}$ crystals, beyond graphene, based on atomically thin films of layered semiconductors such as the family of transition metal dichalcogenides (TMDs) $\left(\mathrm{MX}_{2}, \mathrm{M}=\mathrm{Mo}, \mathrm{W} ; \mathrm{X}=\mathrm{S}, \mathrm{Se}, \mathrm{Te}\right)$ is emerging and has been increasing ever since. The attractiveness of these 2D materials is mainly due to their excellent electrical, mechanical, and optical properties which offer great potential for novel applications, for example, in devices like light emitting diodes (LEDs), ${ }^{1}$ transistors, ${ }^{2}$ sensors, ${ }^{3}$ and photodetectors. ${ }^{4}$ The $\mathrm{MX}_{2}$ monolayers also provide a fundamental requirement for common electronic devices such as novel optoelectronic and photovoltaic applications due to the presence of direct band gap behavior. ${ }^{5-11}$ Moreover, the idea of exploration of a variety of heterostructures from 2D layered TMDs has driven a new platform for modern potential applications such as tunnelling transistors ${ }^{16}$ memory devices, ${ }^{12}$ and ultrathin photodetectors. ${ }^{13}$ Therefore, recently, special attention has been paid to these $2 \mathrm{D}$ crystals both theoretically and experimentally. ${ }^{5,14,15}$ Along these lines, $a b$ initio calculations play a crucial role in introducing new possible 2D layered heterostructures by artificially stacking the monolayer of $\mathrm{MX}_{2}$ for experimentalists and engineers to construct state-of-the-art electronic devices with less effort.

Among the TMDs, $\mathrm{MoS}_{2}$ has been extensively studied, ${ }^{17-19}$ since its properties can be tailored by playing with its environment or even its thickness. For example, drastical changes in its band gap with respect to the adsorbates, strain, interlayer interaction, and charges in neighbouring

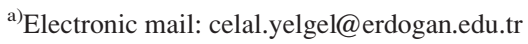

dielectrics were reported. ${ }^{17-19}$ Owing to similar lattice constants, monolayer $\mathrm{MoS}_{2}$ and $\mathrm{WS}_{2}$ are naturally potential substrates to supporting each other. In contrast to planar graphene or monolayer hexagonal boron nitride (h-BN), monolayer $\mathrm{MoS}_{2}$ or $\mathrm{WS}_{2}$ is not single-atom thick since the hexagonally packed layer of Mo or $\mathrm{W}$ atoms is sandwiched between two layers of $\mathrm{S}$ atoms in their monolayer structures. Furthermore, the absence of dangling bonds on their surfaces and charge traps provides great opportunity to create high quality nanoelectronic devices and heterostructures. In their heterostructures, these single layers are mainly stacked together by van der Waals (vdW) interactions. $\mathrm{MoS}_{2}$ and $\mathrm{WS}_{2}$ have an indirect band gap in their bulk form, however, when they are thinned down to a single layer, the band gap becomes direct. In a recent study, it has been shown that monolayer $\mathrm{MoS}_{2}$ strongly emits light because of the $1.8 \mathrm{eV}$ direct band gap at the $\mathrm{K}$ point in the Brillouin zone. ${ }^{20}$ This is also verified by experimental groups using a photoluminescence method. ${ }^{21-23}$ Similarly, the direct band gap of $1.9 \mathrm{eV}$ is obtained for chemical vapor deposition (CVD) islands of monolayer $\mathrm{WS}_{2} .{ }^{24}$ Breaking of inversion symmetry is the reason for transformation to a direct band gap in the monolayer of TMDs. This has also been confirmed experimentally by circularly polarized light experiments that lead to valley polarization effects. ${ }^{25-27}$ TMDs also have large exciton binding energies and strong photoluminescence leading to novel material platforms for optoelectronic applications.

In order to offer functional materials with high performances, one needs to take advantage of 2D heterostructures. For example, a new generation of field-effect-transistors ${ }^{28}$ with a high on-off ratio $\left(>10^{3}\right)$ and a current density of up to $5000 \mathrm{~A} \mathrm{~cm}^{-2}$ has been fabricated using vertically stacked graphene- $\mathrm{MoS}_{2}$-metal heterostructures. Recent studies have reported the successful growth of vertical heterostructures by chemical vapor deposition (CVD), for example, see 
graphene/h-BN, ${ }^{29} \mathrm{MoS}_{2} /$ grapheme $^{30}{ }^{30} \mathrm{MoSe}_{2} /$ grapheme, ${ }^{31}$ $\mathrm{MoS}_{2} / \mathrm{h}-\mathrm{BN},{ }^{32} \mathrm{WS}_{2} / \mathrm{h}-\mathrm{BN},{ }^{33} \mathrm{MX}_{2} / \mathrm{SnS}_{2}(\mathrm{M}=\mathrm{Mo}, \mathrm{W} ; \mathrm{X}=\mathrm{S}$, Se), ${ }^{34}$ and WS2/MoS $2 .{ }^{35}$ The CVD method is one of the best common techniques in order to grow single crystals at a large scale and in a controllable manner, which are very important to fabricating high-quality $\mathrm{MoS}_{2}$ electronic devices. Because of this, researchers have devoted subsequent efforts to the CVD growth of $\mathrm{MoS}_{2}$ on different substrates. ${ }^{37,38}$ One example is the growth of monolayer $\mathrm{MoS}_{2}$ over h-BN by using the seeding method. ${ }^{39}$ Furthermore, the direct growth of monolayer $\mathrm{MoS}_{2}$ has been demonstrated on exfoliated highquality h-BN flakes without using seeding methods, which yield a clean interface between the grown $\mathrm{MoS}_{2}$ and the h-BN substrate. ${ }^{39,40}$ Moreover, the fabrication cost of higher-quality devices is reduced in this method. The advantage of using h-BN as a substrate to grow 2D materials comes from its atomically thin structure without any dangling bonds and charged impurities within it. Furthermore, the properties like being an insulator and a chemically inert system make the h-BN monolayer an ideal substrate for protecting overgrown high quality TMDs for electronic applications and even for improving the device performance. Such examples, for instance, $\mathrm{MoS}_{2}$ deposited over an h-BN substrate, are reported in the literature. ${ }^{17,36}$

The main focus of this study is to characterise novel h-BN/MoS $2 /$ h-BN, h-BN/WS $/$ h-BN, and h-BN/WS $2 / \mathrm{MoS}_{2} /$ h-BN heterostructures by performing extensive firstprinciples calculations. Our aim in these first-principles calculations is to control the magnitude of band gaps in the heterostructures to find a way for developing high-quality durable single- and hetero-layer TMD electronic devices such as optoelectronic devices and field effect transistors. The effects of intercalation of monolayer $\mathrm{MoS}_{2}$, monolayer $\mathrm{WS}_{2}$, and $\mathrm{WS}_{2} / \mathrm{MoS}_{2}$ heterolayer into bilayer $\mathrm{h}-\mathrm{BN}$ and interlayer hybridisation on the structural and electronic properties of monolayer $\mathrm{MoS}_{2}$, monolayer $\mathrm{WS}_{2}$ and $\mathrm{WS}_{2} / \mathrm{MoS}_{2}$ heterolayer are investigated in detail using density functional theory (DFT) with a recently developed non-local van der Waals (vdW) density functional by Vydrov and Van Voorhis (rVV10). ${ }^{41}$ Our computational results suggest that the encapsulated heterostructures, $\mathrm{WS}_{2} / \mathrm{h}-\mathrm{BN}, \mathrm{MoS}_{2} / \mathrm{h}-\mathrm{BN}$, and $\mathrm{WS}_{2} /$ $\mathrm{MoS}_{2} / \mathrm{h}-\mathrm{BN}$, are energetically stable, and the interaction between the layers is weak due to the large interlayer distance, binding energies in the range of physical adsorption, and negligible buckling of the h-BN monolayer indicating van der Waals type interaction. The explanation for interlayer interactions in the encapsulated heterostructures is crucial for proposing and tailoring new technological applications. To the best of our knowledge, there have been no first-principles investigations on the effect of h-BN monolayer on the electronic structure and structural properties of the encapsulated h-BN/WS $/ \mathrm{MoS}_{2} / \mathrm{h}-\mathrm{BN}$ structure. The band structure analysis shows that the direct band gap character of $\mathrm{WS}_{2}$ is still persistent on the $\mathrm{WS}_{2} / \mathrm{h}-\mathrm{BN}$ heterostructure. However, the band gap becomes indirect for monolayer $\mathrm{MoS}_{2}$ and $\mathrm{WS}_{2} / \mathrm{MoS}_{2}$ heterostructure when deposited onto or sandwiched with the h-BN monolayer. It is also worth mentioning that when monolayer $\mathrm{MoS}_{2}$, monolayer $\mathrm{WS}_{2}$ and $\mathrm{WS}_{2} / \mathrm{MoS}_{2}$ heterolayer are deposited onto monolayer h-BN and sandwiched between the h-BN bilayers, the band gap value of $\mathrm{MoS}_{2}$ is reduced to the amount of $0.89 \mathrm{eV}$ in the h-BN/MoS $/$ h-BN heterostructure and $0.14 \mathrm{eV}$ in the $\mathrm{MoS}_{2} /$ h-BN structure. However, the band gap value of $\mathrm{WS}_{2}$ is diminished to the amount of 20 and $710 \mathrm{meV}$ in interfaced and sandwiched structures, respectively. For the $\mathrm{WS}_{2} / \mathrm{MoS}_{2}$ heterolayer structure, the indirect band gap is retained with the band gap values varying from $0.967 \mathrm{eV}$ for $\mathrm{WS}_{2} / \mathrm{MoS}_{2} / \mathrm{h}-$ $\mathrm{BN}$ to $1.110 \mathrm{eV}$ for $\mathrm{h}-\mathrm{BN} / \mathrm{WS}_{2} / \mathrm{MoS}_{2} / \mathrm{h}-\mathrm{BN}$. We further found that the atomically thin flat h-BN monolayers are buckled within the range of $0.01 \AA$ and $0.18 \AA$. This buckling suggests that the h-BN monolayer can be used for gateinsulating materials with minimized interaction between monolayer h-BN and TMDs. Our theoretical investigations offer an improvement in the quality of monolayer $\mathrm{MoS}_{2}$, monolayer $\mathrm{WS}_{2}$, and $\mathrm{WS}_{2} / \mathrm{MoS}_{2}$ heterolayer by using h-BN monolayer and provide band structure engineering of TMDs which is a very useful guide for the construction of TMD based nanoelectronics. From our theoretical predictions, we show that the h-BN monolayer eliminates problematic interfacial defects in TMDs, and can be chosen as an ideal candidate for a high-speed FET.

\section{COMPUTATIONAL METHOD}

All calculations are carried out within the framework of plane-wave density functional theory as embodied in the Quantum ESPRESSO package, ${ }^{42}$ including long-range dispersive interactions with the van der Waals interactioncorrected density functional (DFT/rVV10). ${ }^{41}$ The rVV10 is one of the most popular ones among the DFT + D, $\mathrm{DFT}+\mathrm{D} 2$, and vdW-family in order to effectively describe the nonlocal and long-range nature of the $\mathrm{vdW}$ interaction in the layered materials. This is because it uses the electron density to directly obtain the dispersion interactions, however, DFT $+\mathrm{D}, \mathrm{DFT}+\mathrm{D} 2$, and vdW-family correct the DFT total energy by adding an empirical atom-pairwise interaction correction, parametrized by atomic $\mathrm{C}_{6}$ coefficients. Therefore, this functional is called a non-local correlation functional in which the total exchange-correlation (xc) energy is defined as $E_{x c}=E_{x c}^{0}+E_{c}^{n l}$, where $E_{x c}^{0}$ is the semilocal xc and $E_{c}^{n l}$ is the nonlocal correlation energy discussed in detail in Ref. 41. The rVV10 method gives the most accurate results for intralayer and interlayer lattice constants for 28 layered materials which have been confirmed from experimental results in Ref. 43. The electron-ion interaction is described by ultrasoft pseudopotentials. ${ }^{44}$ A plane-wave basis set with a kinetic energy cutoff of 60 Ryd is adopted to expand the single-particle Kohn-Sham orbitals. Brillouin zone sampling of electronic states is approximated by using the sets of special k-points corresponding to the $(36 \times 36 \times 1)$ Monkhorst-Park mesh for monolayer $\mathrm{MoS}_{2}$, monolayer $\mathrm{WS}_{2}$, and $\mathrm{WS}_{2} / \mathrm{MoS}_{2}$ heterolayer and $(12 \times 12 \times 1)$ Monkhorst-Park mesh for proposed heterostructures. ${ }^{45}$ To hinder spurious interactions between two supercells, a vacuum buffer space of $10 \AA$ is inserted in the $z$ direction which is perpendicular to the plane of monolayer and heterolayers. All the cell parameters and the atomic coordinates are relaxed until the maximum Hellmann- 
TABLE I. The calculated structural parameters such as a, lattice constant; $\mathrm{d}_{M o-S}, \mathrm{~d}_{W-S}$, and $\mathrm{d}_{B-N}$, Mo-S, W-S, and B-N bond lengths; $\mathrm{E}_{b}$, binding energies; $\mathrm{h}_{M o-M o}$ and $\mathrm{h}_{W-W}$, vertical distance of two Mo atoms and two $\mathrm{W}$ atoms and $\mathrm{E}_{g}$, computed band gap values.

\begin{tabular}{|c|c|c|c|c|c|c|}
\hline System & $\mathrm{a}(\AA)$ & $\mathrm{d}_{M o-S} / \mathrm{d}_{W-S}(\AA)$ & $\mathrm{d}_{B-N}(\AA)$ & $\mathrm{E}_{b}(\mathrm{meV} / \mathrm{atom})$ & $\mathrm{h}_{M o-M o} / \mathrm{h}_{W-W}(\AA)$ & $\mathrm{E}_{g}(\mathrm{eV})$ \\
\hline Monolayer $\mathrm{MoS}_{2}$ & 3.185 & $2.418 /-$ & & & & 1.850 \\
\hline Monolayer $\mathrm{WS}_{2}$ & 3.173 & $-/ 2.410$ & & & & 1.940 \\
\hline Monolayer h-BN & 2.510 & & 1.452 & & & 4.480 \\
\hline Bilayer $\mathrm{MoS}_{2}$ & 3.185 & $2.418 /-$ & & 59.333 & $6.064 /-$ & 1.142 (indirect) \\
\hline Bilayer $\mathrm{WS}_{2}$ & 3.173 & $-/ 2.410$ & & 59.833 & $-/ 5.845$ & 1.051 (indirect) \\
\hline Bulk $\mathrm{MoS}_{2}$ & 3.185 & $2.418 /-$ & & & $6.247 /-$ & 0.975 (indirect) \\
\hline Bulk WS $\mathrm{W}_{2}$ & 3.173 & $-/ 2.410$ & & & $-/ 6.247$ & 0.895 (indirect) \\
\hline
\end{tabular}

Feynman force acting on each atoms is smaller than $0.03 \mathrm{eVA}^{-1}$. The total energy convergence is set to less than $10^{-6} \mathrm{eV}$ in our calculations. The equilibrium geometries are fully optimised. To populate the electron state in the selfconsistent calculations, the Methfessel Paxton scheme ${ }^{46}$ is used with a smearing width of $0.05 \mathrm{eV}$.

\section{RESULTS AND DISCUSSION}

First, the lattice constants of single layer $\mathrm{MoS}_{2}, \mathrm{WS}_{2}$, and $\mathrm{h}-\mathrm{BN}$ are calculated to be $a=3.185 \AA$, 3.173 $\AA$, and $2.510 \AA$, respectively, as summarised in Table I. This is in reasonable agreement with previous results. ${ }^{4,48}$ Even though there is a quite difference between the lattice constants of $\mathrm{MoS}_{2}$ or $\mathrm{WS}_{2}$ and monolayer h-BN, all of them have a hexagonal structure, as shown in Figs. 1(a)-1(c). In order to set up an appropriate unit cell for $\mathrm{MoS}_{2} / \mathrm{h}-\mathrm{BN}$ and $\mathrm{WS}_{2} /$ h-BN, and their sandwiched structures, we use $4 \times 4 \mathrm{MoS}_{2}$ and $\mathrm{WS}_{2}$ monolayers to match the $5 \times 5 \mathrm{~h}$-BN monolayer which results in an almost perfect match with the lattice mismatches of $\sim 1.6 \%$ and $\sim 1.1 \%$ for $\mathrm{MoS}_{2} / \mathrm{h}-\mathrm{BN}$ and $\mathrm{WS}_{2} /$ h-BN heterostructures, respectively. These lattice mismatches are considerably small compared with previously studied heterostructures. ${ }^{49,50}$ We calculate Mo-S, W-S, and B-N bond lengths as $2.418 \AA, 2.410 \AA$, and $1.452 \AA$, respectively, which are consistent with the reported values in Refs. 47, 51, and 52.

Next, we construct $\mathrm{MoS}_{2}$ and $\mathrm{WS}_{2}$ bilayer and $\mathrm{WS}_{2}$ / $\mathrm{MoS}_{2}$ heterolayer systems as shown in Figs. 2(a)-2(c). For the bilayer and heterolayer systems, two different stacking orders, $\mathrm{AA}$ and $\mathrm{AB}$, are considered. In $\mathrm{AB}$ stacking, the $\mathrm{S}$ atoms in the top layer are directly aligned above the Mo (W) atoms in the bottom layer, while AA stacking refers to Mo or $\mathrm{W}(\mathrm{S})$ atoms on the top layer right above the same atoms of the adjacent layer. Our test calculations suggest that $A B$ stacking is the most energetically stable configuration. For the $\mathrm{WS}_{2} / \mathrm{MoS}_{2}$ heterostructure, we simply use the primitive cells of $\mathrm{MoS}_{2}$ and $\mathrm{WS}_{2}$ with negligible strain since the lattice mismatch between them is much smaller $(0.5 \%)$ than $\mathrm{MoS}_{2} /$
h-BN. The vertical distance between two Mo atoms, two W atoms, and Mo and $\mathrm{W}$ atoms in adjacent layers is found to be $6.064 \AA$, $5.845 \AA$, and $6.148 \AA$ for bilayer $\mathrm{MoS}_{2}$, bilayer $\mathrm{WS}_{2}$, and $\mathrm{WS}_{2} / \mathrm{MoS}_{2}$ heterolayer, respectively, which indicates a van der Waals type interaction between the layers. These distances have excellent agreement with reported distances in Ref. 53. Moreover, the similar interlayer distances between two Mo and two $\mathrm{W}$ atoms in adjacent layers in bulk $\mathrm{MoS}_{2}$ and $\mathrm{WS}_{2}$ are calculated as $6.247 \AA$, which are in very good agreement with the experimental value [6.220 $\AA$ (Refs. 53 and 54)], hence the vdW-corrected functional is reliable for these calculations. We calculate the total energy difference between the $\mathrm{AA}$ and $\mathrm{AB}$ stacking orders for the $\mathrm{WS}_{2}$ / $\mathrm{MoS}_{2}$ heterolayer; the AB stacking is energetically favored with an energy difference of $0.069 \mathrm{eV} /$ cell. The binding energies $\left(\mathrm{E}_{b}\right)$ between the layers for bilayer $\mathrm{MoS}_{2}$ and $\mathrm{WS}_{2}$, and heterolayer $\mathrm{WS}_{2} / \mathrm{MoS}_{2}$ are defined as

$$
\begin{gathered}
E_{b}=\left[E\left(M o S_{2} \text { bilayer }\right)-2 \times E\left(M o S_{2} \text { monolayer }\right)\right] / N, \\
E_{b}=\left[E\left(W S_{2} \text { bilayer }\right)-2 \times E\left(W S_{2} \text { monolayer }\right)\right] / N, \\
E_{b}=\left[E\left(W S_{2} / M o S_{2} \text { heterobilayer }\right)-E\left(M o S_{2} \text { monolayer }\right)\right. \\
\left.-E\left(W S_{2} \text { monolayer }\right)\right] / N,
\end{gathered}
$$

where $\mathrm{E}\left(\mathrm{MoS}_{2}\right.$ bilayer $), \mathrm{E}\left(\mathrm{WS}_{2}\right.$ bilayer $)$, and $\mathrm{E}\left(\mathrm{WS}_{2} / \mathrm{MoS}_{2}\right.$ heterobilayer) are the total energies of the $\mathrm{MoS}_{2}$ bilayer, $\mathrm{WS}_{2}$ bilayer, and $\mathrm{WS}_{2} / \mathrm{MoS}_{2}$ heterobilayer, respectively; $\mathrm{E}_{\mathrm{MoS}_{2}}$ and $\mathrm{E}_{W S_{2}}$ are the total energies of $\mathrm{MoS}_{2}$ and $\mathrm{WS}_{2}$ monolayers, respectively; and $N$ is the total number of atoms in the supercell. The binding energies per atom are listed in Tables I and II. We found almost no differences between binding energies for bilayer $\mathrm{MoS}_{2}$ and $\mathrm{WS}_{2}$. However, when the $\mathrm{MoS}_{2}$ monolayer is stacked on the $\mathrm{WS}_{2}$ monolayer, the binding energy is increased by an amount of $10 \mathrm{meV}$, making the interlayer interaction slightly stronger. We further found a good agreement with available values reported in Refs. 54 and 55. This is another indication that the interlayer bonding of $\mathrm{MoS}_{2}$ and $\mathrm{WS}_{2}$ is weak van der Waals interactions. We (a)

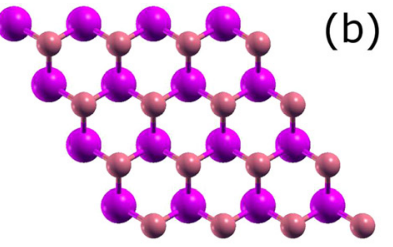

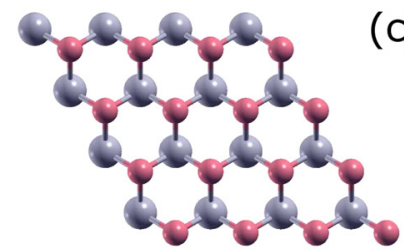

(c)

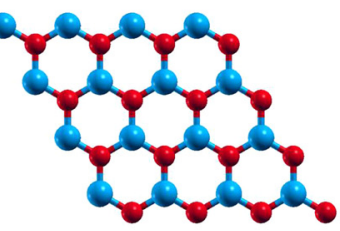

FIG. 1. Optimised geometric structures of (a) monolayer $\mathrm{MoS}_{2}$, (b) monolayer $\mathrm{WS}_{2}$, and (c) monolayer h-BN. The purple, grey, pink, red, and blue balls represent Mo, W, S, N, and B atoms, respectively. 
(a)

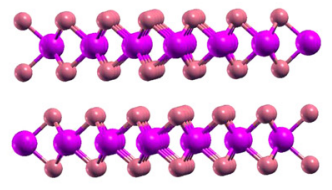

(b)

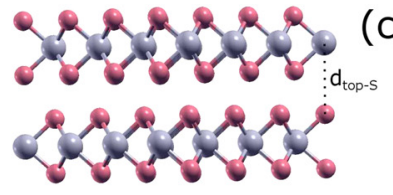

(c)

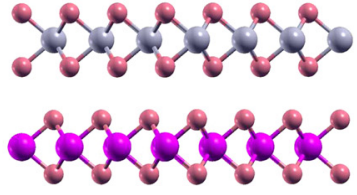

FIG. 2. Optimised geometric structures of (a) bilayer $\mathrm{MoS}_{2}$, (b) bilayer $\mathrm{WS}_{2}$, and (c) $\mathrm{WS}_{2} / \mathrm{MoS}_{2}$ heterostructure. The purple, grey, pink, red, and blue balls represent Mo, W, S, N, and B atoms, respectively. also notice that there are no significant changes in Mo-S and W-S bond lengths when the lattice constant of either $\mathrm{MoS}_{2}$ or $\mathrm{WS}_{2}$ is used in the $\mathrm{WS}_{2} / \mathrm{MoS}_{2}$ heterostructure.

To obtain the equilibrium geometry of $\mathrm{MoS}_{2} / \mathrm{h}-\mathrm{BN}$, $\mathrm{WS}_{2} / \mathrm{h}-\mathrm{BN}$, and $\mathrm{WS}_{2} / \mathrm{MoS}_{2} / \mathrm{h}-\mathrm{BN}$ heterostructures and their sandwiched structures, we optimised the heterostructures using several starting positions of $\mathrm{MoS}_{2}, \mathrm{WS}_{2}$, and $\mathrm{WS}_{2} /$ $\mathrm{MoS}_{2}$ subject to the h-BN one. After optimisation calculations, we found the most stable configuration with one Mo (W) atom on top of the $\mathrm{B}$ atom and the $\mathrm{S}$ atom near the $\mathrm{N}$ atom site on the bottom layer of the heterostructures, as shown in Figs. 3(a)-3(f). For sandwiched structures, different stacking orders are considered by rotating the top monolayer h-BN. After our total energy calculations, we found the total energy differences being less than $9.685 \mathrm{meV}$ between different types of their stacking orders. Therefore, the ABA stacking is used in all sandwiched structures. The structural parameters are summarised in Table II for the heterostructures. The interlayer spacing between monolayer h-BN and the topmost $\mathrm{S}$ atom in $\mathrm{MoS}_{2}$ and $\mathrm{WS}_{2}$ is estimated as $3.451 \AA$ and $3.425 \AA$ for $\mathrm{MoS}_{2} / \mathrm{h}-\mathrm{BN}$ and $\mathrm{WS}_{2} / \mathrm{h}$-BN heterostructures, respectively. For $\mathrm{MoS}_{2} / \mathrm{h}-\mathrm{BN}$ heterostructures, the Mo-S and B-N bond lengths are found to be $2.420 \AA$ and $1.470 \AA$, respectively, which represent $1.4 \%$ stretching of the monolayer h-BN substrate. The substrate is also slightly buckled with the amount of $0.18 \AA$. In the $\mathrm{WS}_{2} / \mathrm{h}$-BN heterostructure, the vertical distance of the topmost $\mathrm{S}$ atoms in $\mathrm{WS}_{2}$ from monolayer h-BN is found to be $3.425 \AA$ together with $\mathrm{B}-\mathrm{N}$ and $\mathrm{W}-\mathrm{S}$ bond lengths of $1.470 \AA$ and $2.410 \AA$, respectively. Interestingly, while the monolayer h-BN is buckled in the $\mathrm{MoS}_{2} / \mathrm{h}$-BN heterostructure, the monolayer h-BN is almost flat with the buckling of $0.03 \AA$ in $\mathrm{WS}_{2} / \mathrm{h}$ $\mathrm{BN}$. When the $\mathrm{WS}_{2} / \mathrm{MoS}_{2}$ heterostructure is deposited on monolayer h-BN, the interfacial distance between $\mathrm{MoS}_{2}$ and $\mathrm{WS}_{2}$ monolayers is increased from $6.148 \AA$ to $6.303 \AA$ representing the weakening the interaction between the layers. The interlayer spacing between monolayer h-BN and the topmost $\mathrm{S}$ atoms in $\mathrm{MoS}_{2}$ is found to be $3.512 \AA$ with the negligible rumpling of $0.01 \AA$ in the h-BN monolayer. The computed binding energies for $\mathrm{MoS}_{2} / \mathrm{h}-\mathrm{BN}, \mathrm{WS}_{2} / \mathrm{h}-\mathrm{BN}$, and $\mathrm{WS}_{2} / \mathrm{MoS}_{2} / \mathrm{h}-\mathrm{BN}$ heterostructures are listed in Table II. The binding energy of these heterostructures is defined as

$$
\begin{gathered}
\left.E_{b}=\left[E_{M o S_{2} / h-B N}-E_{M o S_{2}}-E_{h-B N}\right)\right] / N, \\
\left.E_{b}=\left[E_{W S_{2} / h-B N}-E_{W S_{2}}-E_{h-B N}\right)\right] / N, \\
\left.E_{b}=\left[E_{W S_{2} / M o S_{2} / h-B N}-E_{M o S_{2}}-E_{W S_{2}}-E_{h-B N}\right)\right] / N,
\end{gathered}
$$

where $\mathrm{E}_{M o S_{2} / h-B N}, \mathrm{E}_{W S_{2} / h-B N}$, and $\mathrm{E}_{W S_{2} / M o S_{2} / h-B N}$ are the total energies of the $\mathrm{MoS}_{2} / \mathrm{h}-\mathrm{BN}, \mathrm{WS}_{2} / \mathrm{h}-\mathrm{BN}$, and $\mathrm{WS}_{2} /$ $\mathrm{MoS}_{2} / \mathrm{h}-\mathrm{BN}$ heterostructures, respectively; $\mathrm{E}_{\mathrm{MoS}_{2}}, \mathrm{E}_{W S_{2}}$, and $\mathrm{E}_{h-B N}$ are the total energies of $\mathrm{MoS}_{2}, \mathrm{WS}_{2}$, and h-BN monolayers, respectively; and $N$ is the total number of atoms in the heterostructure. For sandwiched structures, the calculated equilibrium interlayer distances are $3.566 \AA, 3.583 \AA$, and $3.476 \AA$ for h-BN/MoS $/$ h-BN, h-BN/WS $2 /$ h-BN, and h-BN/ $\mathrm{WS}_{2} / \mathrm{MoS}_{2} / \mathrm{h}-\mathrm{BN}$ systems, respectively. However, for the h$\mathrm{BN} / \mathrm{WS}_{2} / \mathrm{MoS}_{2} / \mathrm{h}-\mathrm{BN}$ system, we found that the interlayer distance between the top h-BN monolayer and the topmost $\mathrm{S}$ atoms in the $\mathrm{WS}_{2}$ monolayer is $3.476 \AA$, which is smaller than that of the distance between the bottom h-BN monolayer and the topmost $\mathrm{S}$ atoms in the $\mathrm{MoS}_{2}$ monolayer. For all sandwiched structures, a negligible buckling of $0.02 \AA$ in both top and bottom layers of h-BN is induced by the $\mathrm{MoS}_{2}$, $\mathrm{WS}_{2}$, and $\mathrm{WS}_{2} / \mathrm{MoS}_{2}$ layers. To analyse the stabilities of these sandwiched heterostructures, the binding energy of the heterostructure is defined with the following equations:

$$
\begin{gathered}
\left.E_{b}=\left[E_{h-B N / M o S_{2} / h-B N}-E_{M o S_{2}}-2 \times E_{h-B N}\right)\right] / N \\
\left.E_{b}=\left[E_{h-B N / W S_{2} / h-B N}-E_{W S_{2}}-2 \times E_{h-B N}\right)\right] / N \\
\left.E_{b}=\left[E_{h-B N / W S_{2} / M o S_{2} / h-B N}-E_{M o S_{2}}-E_{W S_{2}}-2 \times E_{h-B N}\right)\right] / N,
\end{gathered}
$$

where $\quad \mathrm{E}_{h-B N / M o S_{2} / h-B N}, \quad \mathrm{E}_{h-B N / W S_{2} / h-B N}, \quad$ and

\begin{tabular}{|c|c|c|c|c|c|}
\hline System & $\mathrm{d}_{M o-S} / \mathrm{d}_{W-S}(\AA)$ & $\mathrm{d}_{B-N} / \Delta_{B N}(\AA)$ & $\mathrm{E}_{b}(\mathrm{meV} /$ atom $)$ & $\mathrm{h}_{0} / \mathrm{h}_{M o-W}$ & $\mathrm{E}_{g}(\mathrm{eV})$ \\
\hline $\mathrm{WS}_{2} / \mathrm{MoS}_{2}$ & $2.418 / 2.410$ & & 49.833 & $-/ 6.148$ & 0.907 (indirect) \\
\hline $\mathrm{MoS}_{2} / \mathrm{h}-\mathrm{BN}$ & $2.420 /-$ & $1.470 / 0.18$ & 41.225 & $3.451 /-$ & 1.710 (indirect) \\
\hline $\mathrm{WS}_{2} / \mathrm{h}-\mathrm{BN}$ & $-/ 2.410$ & $1.470 / 0.03$ & 42.531 & $3.425 /-$ & 1.920 \\
\hline $\mathrm{WS}_{2} / \mathrm{MoS}_{2} / \mathrm{h}-\mathrm{BN}$ & $2.418 / 2.410$ & $1.460 / 0.01$ & 46.322 & $3.512 / 6.303$ & 0.967 (indirect) \\
\hline h-BN/MoS $2 /$ h-BN & $2.417 /-$ & $1.471 / 0.02$ & 53.101 & $3.566 /-$ & 0.960 (indirect) \\
\hline h-BN/WS $2 /$ h-BN & $-/ 2.410$ & $1.465 / 0.02$ & 54.081 & $3.583 /-$ & 1.230 (indirect) \\
\hline h-BN/WS $/ \mathrm{MoS}_{2} / \mathrm{h}-\mathrm{BN}$ & $2.415 / 2.410$ & $1.471 / 0.03$ & 55.219 & $3.476 / 6.171$ & 1.110 (indirect) \\
\hline
\end{tabular}
$\mathrm{E}_{h-B N / W S_{2} / M O S_{2} / h-B N}$ are the total energies of the h-BN/ $\mathrm{MoS}_{2} / \mathrm{h}-\mathrm{BN}, \mathrm{h}-\mathrm{BN} / \mathrm{WS}_{2} / \mathrm{h}-\mathrm{BN}$, and h-BN/WS $/ \mathrm{MoS}_{2} / \mathrm{h}-\mathrm{BN}$

TABLE II. The calculated structural parameters such as a, lattice constant; $\mathrm{d}_{M o-S}, \mathrm{~d}_{W-S}$, and $\mathrm{d}_{B-N}$, Mo-S, W-S, and B-N bond lengths; $\Delta_{B N}$, buckling of the monolayer h-BN; $\mathrm{E}_{b}$, binding energies; $\mathrm{h}_{0}$, interlayer spacing between monolayer $\mathrm{h}-\mathrm{BN}$ and the topmost $\mathrm{S}$ atom in $\mathrm{MoS}_{2}$ and $\mathrm{WS}_{2} ; \mathrm{h}_{M o-W}$, vertical distance between $\mathrm{Mo}$ and $\mathrm{W}$ atoms and $\mathrm{E}_{g}$, computed band gap values. 
(a)

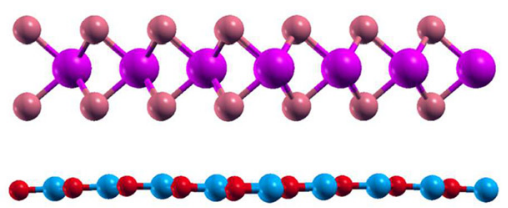

(b)

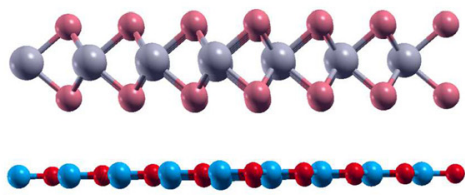

(c)
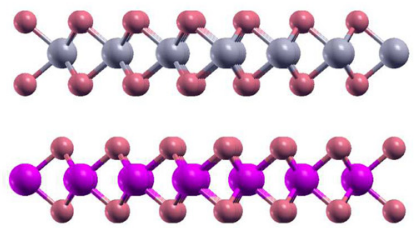

$0-00-0-c 0-0-0-0-00-00$ (d)

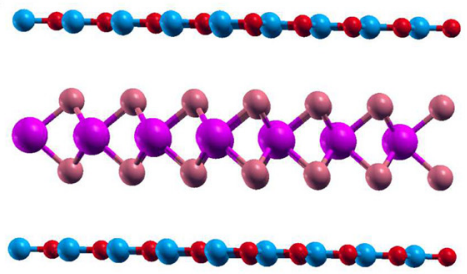

(e)

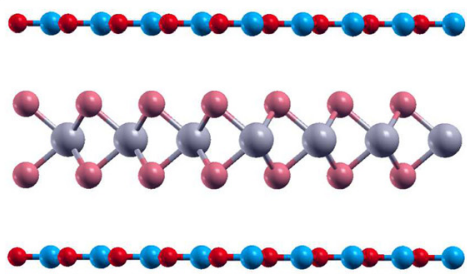

(f)

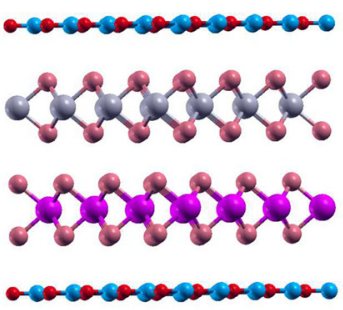

FIG. 3. Optimised geometric structures of (a) $\mathrm{MoS}_{2} / \mathrm{h}-\mathrm{BN}$, (b) $\mathrm{WS}_{2} / \mathrm{h}-\mathrm{BN}$, (c) $\mathrm{WS}_{2} / \mathrm{MoS}_{2} / \mathrm{h}-\mathrm{BN}$, (d) h-BN/MoS $2 / \mathrm{h}-$ $\mathrm{BN}$ h-BN, (e) h-BN/WS $2 / \mathrm{h}-\mathrm{BN}$, and (f) h-BN/WS $/ \mathrm{WoS}_{2} / \mathrm{h}-\mathrm{BN}$ heterostructures. The purple, grey, pink, red, and blue balls represent Mo, W, S, N, and $\mathrm{B}$ atoms, respectively. heterostructures, respectively; $\mathrm{E}_{\mathrm{MoS}_{2}}, \mathrm{E}_{W S_{2}}$, and $\mathrm{E}_{h-B N}$ are the total energies of $\mathrm{MoS}_{2}, \mathrm{WS}_{2}$, and h-BN monolayer, respectively; and $N$ is the total number of atoms in the heterostructure. We obtained a negative binding energy for all heterostructures suggesting that the formation of heterostructures is an exothermic process from the thermodynamic point of view. This is also an indication that these heterostructures are energetically favorable. As summarised in Table II, the smaller binding energy represents the strongest binding between the layers in the heterostructures. The values of $\mathrm{E}_{b}$ are in the range of physical adsorption which suggests weak $\mathrm{vdW}$ interactions between the layers. Our results also suggest that the interlayer interaction in $\mathrm{MoS}_{2} / \mathrm{h}-\mathrm{BN}, \mathrm{WS}_{2} / \mathrm{h}-\mathrm{BN}$, and $\mathrm{WS}_{2} / \mathrm{MoS}_{2} / \mathrm{h}-\mathrm{BN}$ heterostructures is slightly stronger than that in the sandwiched structures. From the present results, we suggest that $\mathrm{MoS}_{2}, \mathrm{WS}_{2}$, and $\mathrm{WS}_{2} / \mathrm{MoS}_{2}$ layers interact very weakly with the h-BN monolayer due to the large distance between the TMD layers and the h-BN monolayer, and a small rumpling in h-BN. This also leads to significantly weak electronic coupling between the layers and the h-BN monolayer.

After the determination of the structural stability of h$\mathrm{BN} / \mathrm{MoS}_{2} / \mathrm{h}-\mathrm{BN}, \mathrm{h}-\mathrm{BN} / \mathrm{WS}_{2} / \mathrm{h}-\mathrm{BN}$, and h-BN/WS $/ \mathrm{MoS}_{2} / \mathrm{h}-$ $\mathrm{BN}$ heterostructures, we now present the electronic band structures of these heterostructures. Figures 4(a)-4(c) shows the electronic structure of monolayer $\mathrm{MoS}_{2}, \mathrm{WS}_{2}$, and h-BN. Our vdW-DF calculations indicate that both monolayer $\mathrm{MoS}_{2}$ and monolayer $\mathrm{WS}_{2}$ have direct band gaps of $1.850 \mathrm{eV}$ and $1.940 \mathrm{eV}$, respectively, consistent with the previously reported studies. ${ }^{20,24,49}$ As shown in Fig. 4(c), we found a large band gap of $4.480 \mathrm{eV}$ for monolayer h-BN with vdWDF calculations, closer to the experimental value. ${ }^{56}$ Because of this large band gap, monolayer h-BN is considered as the best candidate for enhancing the band gap in $\mathrm{vdW}$ heterostructures. For the $\mathrm{WS}_{2} / \mathrm{MoS}_{2}$ heterostructure, the indirect band gap of $0.907 \mathrm{eV}$ is obtained as shown in Fig. 4(f). This indirect band gap is due to the interlayer coupling between monolayer $\mathrm{MoS}_{2}$ and monolayer $\mathrm{WS}_{2}$. The band gap is also considerably smaller than that of both monolayer $\mathrm{MoS}_{2}$ and $\mathrm{WS}_{2}$. Similarly, the in-direct band gaps of $0.975 \mathrm{eV}$ and $0.895 \mathrm{eV}$ are observed in bulk $\mathrm{MoS}_{2}$ and bulk $\mathrm{WS}_{2}$, respectively, in good agreement with experimental values. ${ }^{57,58}$ When a second layer of $\mathrm{MoS}_{2}$ and $\mathrm{WS}_{2}$ is put on the monolayer $\mathrm{MoS}_{2}$ and $\mathrm{WS}_{2}$, the direct band gap is transformed into the indirect band gap. As presented in Figs. 4(d) and 4(e), our calculated gap values are $1.142 \mathrm{eV}$ and $1.051 \mathrm{eV}$ for bilayer $\mathrm{MoS}_{2}$ and $\mathrm{WS}_{2}$, respectively.

After depositing $\mathrm{MoS}_{2}$ on monolayer h-BN, the direct band gap nature of $\mathrm{MoS}_{2}$ changes to an indirect band gap. As shown in Fig. 5(a), the calculated direct gap at the $\mathbf{K}$ point and the indirect gap are $1.805 \mathrm{eV}$ and $1.710 \mathrm{eV}$, respectively. When another monolayer h-BN is added on top of $\mathrm{MoS}_{2} / \mathrm{h}-\mathrm{BN}$ heterostructure, we notice that the indirect band gap is preserved with the value of $0.960 \mathrm{eV}$, as presented in Fig. 5(b). However, we found that the direct band gap at the $\mathbf{K}$ point is decreased to $1.112 \mathrm{eV}$, and the indirect band gap value is also reduced to $0.960 \mathrm{eV}$. In both interfaced and sandwiched structures, the valence band maximum (VBM) at the $\mathbf{K}$ point is smaller than the valence band edge at the $\Gamma$ point. When the $\mathrm{WS}_{2}$ layer is stacked on the h-BN monolayer, the direct band gap remained with the value of $1.920 \mathrm{eV}$, as indicated in Fig. 6(a). However, the direct band gap is transformed into an indirect band gap with the value of $1.230 \mathrm{eV}$ [shown in Fig. 6(b)], when the $\mathrm{WS}_{2}$ monolayer is intercalated into bilayer h-BN. It is worth mentioning that the direct band gap value of $\mathrm{WS}_{2}$ is slightly decreased with 
(a)

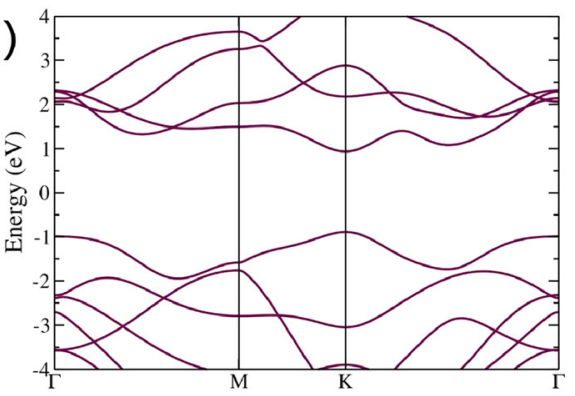

(b)

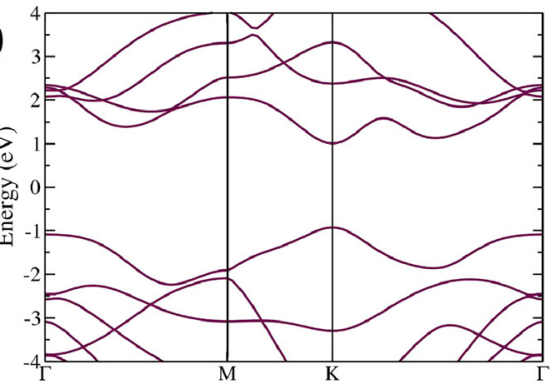

(c)

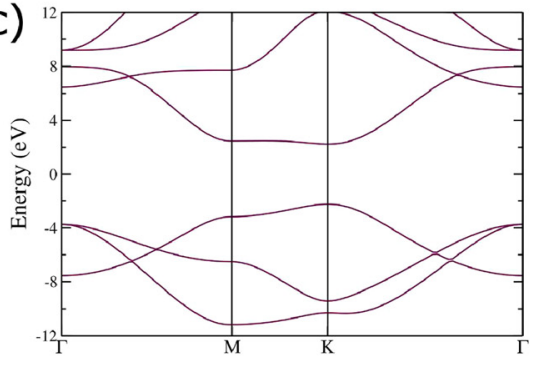

(d)

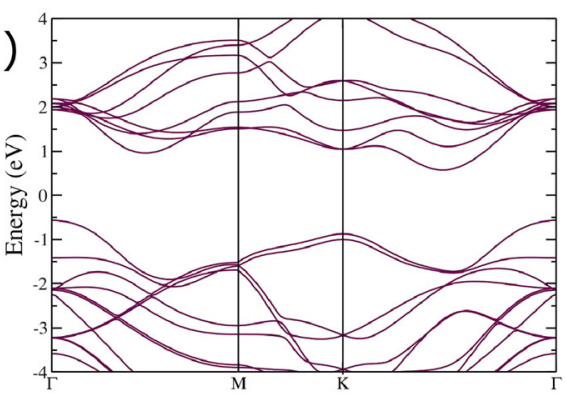

(e)

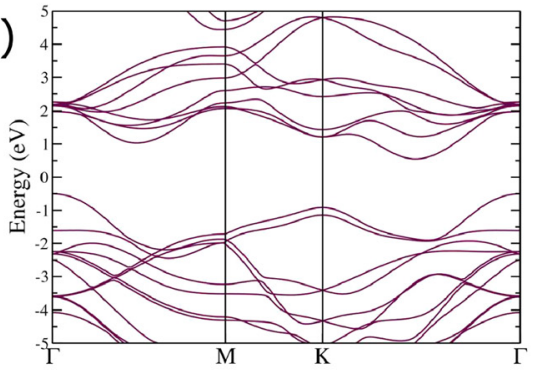

FIG. 4. Band structures of monolayer (a) $\mathrm{MoS}_{2}$, (b) $\mathrm{WS}_{2}$, (c) h-BN, (d) bilayer $\mathrm{MoS}_{2}$, (e) bilayer $\mathrm{WS}_{2}$, and (f) $\mathrm{WS}_{2} /$ $\mathrm{MoS}_{2}$ heterolayer. The Fermi level is set to zero.

(f)

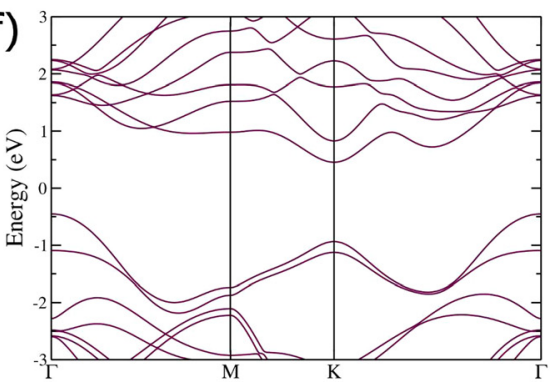

(a)

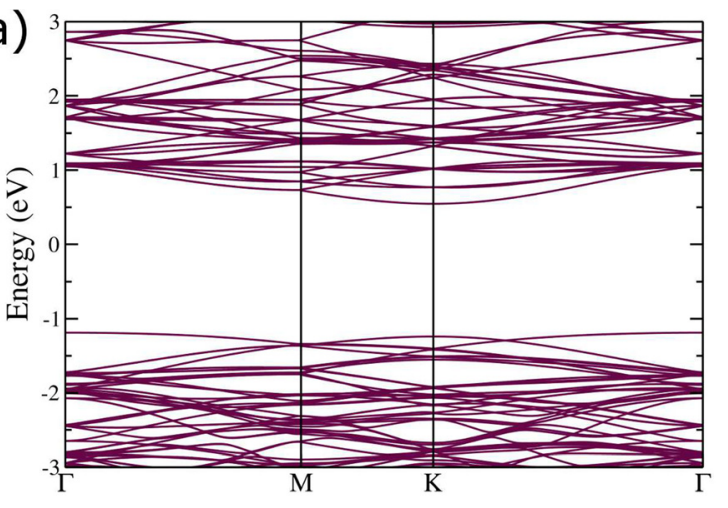

(b)

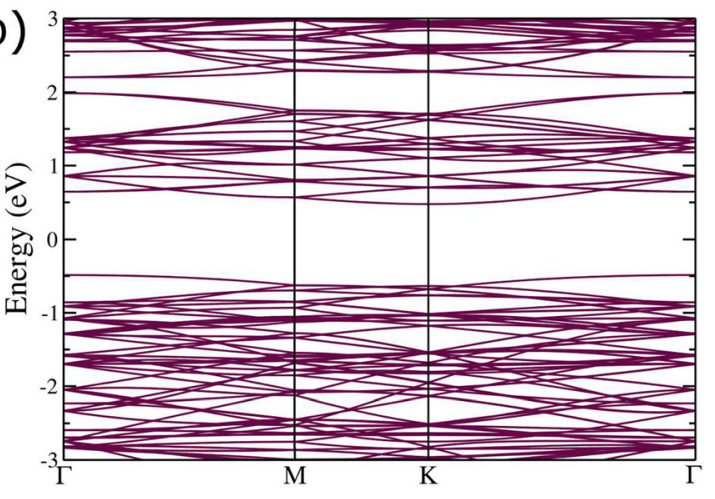

FIG. 5. Band structures of (a) $\mathrm{MoS}_{2} / \mathrm{h}-\mathrm{BN}$ and (b) h-BN/MoS $2 / \mathrm{h}-\mathrm{BN}$ heterostructures. The Fermi level is set to zero. (a)

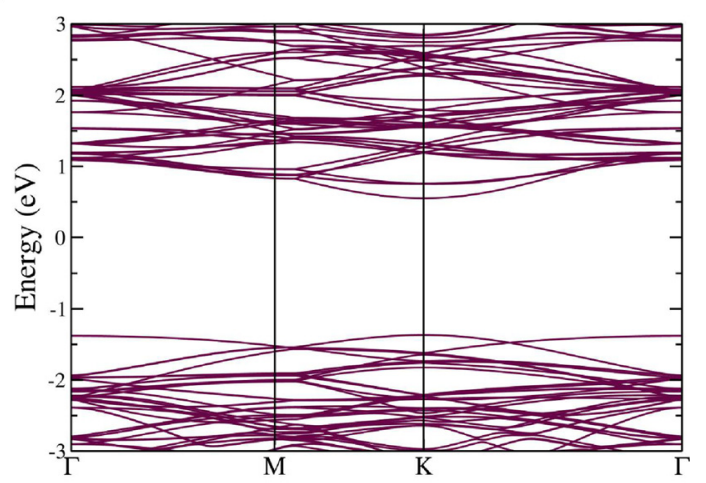

(b)

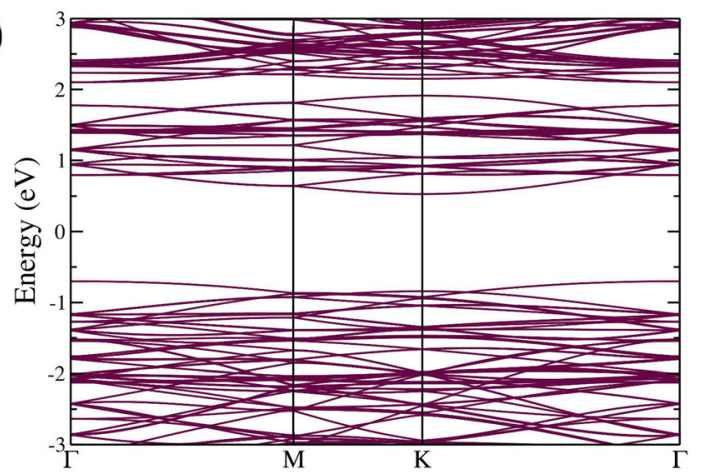

FIG. 6. Band structures of (a) $\mathrm{WS}_{2} / \mathrm{h}-\mathrm{BN}$ and (b) h-BN/WS $/ \mathrm{h}-\mathrm{BN}$ heterostructures. The Fermi level is set to zero. 


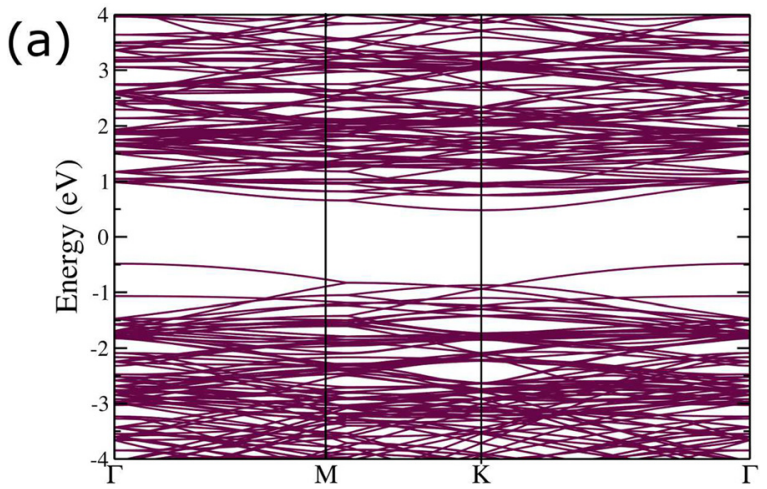

(b)

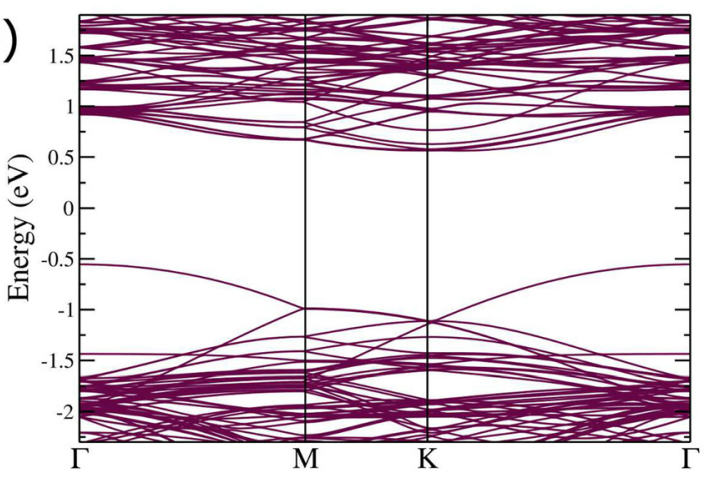

FIG. 7. Band structures of (a) $\mathrm{WS}_{2} / \mathrm{MoS}_{2} / \mathrm{h}-\mathrm{BN}$ and (b) h-BN/WS $/ \mathrm{WoS}_{2} /$ h-BN heterostructures. The Fermi level is set to zero.

the amount of $20 \mathrm{meV}$ when compared with the $\mathrm{WS}_{2}$ heterostructure, exhibiting no noticeable covalent bonding of the layers. The calculated band structures of $\mathrm{WS}_{2} / \mathrm{MoS}_{2} / \mathrm{h}-\mathrm{BN}$ and $\mathrm{h}-\mathrm{BN} / \mathrm{WS}_{2} / \mathrm{MoS}_{2} / \mathrm{h}-\mathrm{BN}$ heterostructures are presented in Figs. 7(a) and 7(b). They are both indirect band gap semiconductors with the value of $0.967 \mathrm{eV}$ and $1.110 \mathrm{eV}$, respectively. It is clear that the indirect band gap of $\mathrm{WS}_{2} / \mathrm{MoS}_{2}$ is reduced to $0.967 \mathrm{eV}$ when the $\mathrm{WS}_{2} / \mathrm{MoS}_{2}$ heterostructure is deposited on monolayer h-BN. However, the indirect band gap for the $\mathrm{WS}_{2} / \mathrm{MoS}_{2}$ heterostructure varies from $0.907 \mathrm{eV}$ to $1.110 \mathrm{eV}$ when inserting the $\mathrm{WS}_{2} / \mathrm{MoS}_{2}$ heterostructure into bilayer h-BN implying the protection of its intrinsic properties.

To further analyse the electronic interaction between monolayer $\mathrm{MoS}_{2}, \mathrm{WS}_{2}$, and h-BN, we calculate the total density of states (TDOS) and partial density of states (PDOS) including the d-electrons of the transition metals and the p-electrons of chalcogen, boron, and nitrogen atoms. Figures 8(a) and 8(b) show the TDOS and the PDOS of the $\mathrm{WS}_{2} / \mathrm{MoS}_{2}$ heterostructure. The valence band maximum
(VBM) is contributed by d orbitals of both $\mathrm{W}$ and Mo atoms and the $\mathrm{p}$ orbital of the $\mathrm{S}$ atom, while the conduction band minimum (CBM) is mainly dominated by the d orbitals of Mo atoms. For the $\mathrm{MoS}_{2} / \mathrm{h}-\mathrm{BN}$ system, PDOS analysis reveals that the states below the Fermi level are slightly dominated by only $\mathrm{p}$ orbitals of $\mathrm{N}$ atoms [see Figs. 9(a) and 9(c)], while there is almost no contribution from h-BN monolayer above the Fermi level. As clearly shown in Fig. 9(c), most states in both occupied and unoccupied regions arose from Mo d-electrons for $\mathrm{MoS}_{2} / \mathrm{h}$-BN. Intercalating a monolayer $\mathrm{MoS}_{2}$ into a bilayer $\mathrm{h}-\mathrm{BN}$ increases the negligible contribution of the $\mathrm{p}$ orbital of $\mathrm{B}$ atoms above the Fermi level, while vanishing the contribution of $\mathrm{p}$ orbitals of $\mathrm{N}$ atoms below the Fermi level as presented in Figs. 9(b) and 9(d). Additionally, new peaks are generated at $\mathrm{E}_{f}+0.725 \mathrm{eV}$ in the unoccupied region, at $\mathrm{E}_{f}-0.725 \mathrm{eV}$ and $\mathrm{E}_{f}-1.115 \mathrm{eV}$ in the occupied region, which are mostly dominated by the $\mathrm{d}$ electrons of Mo atoms. Furthermore, both peaks at $\mathrm{E}_{f}+1.051 \mathrm{eV}$ and $\mathrm{E}_{f}-2.015 \mathrm{eV}$ in $\mathrm{MoS}_{2} / \mathrm{h}-\mathrm{BN}$ structures are shifted when another h-BN monolayer is added to the $\mathrm{MoS}_{2} /$ $\mathrm{h}-\mathrm{BN}$ heterolayer. The PDOS and TDOS for $\mathrm{WS}_{2} / \mathrm{h}-\mathrm{BN}$ and h-BN/WS $/$ h-BN heterostructures are shown in Figs. 10(a)-10(d). The conduction band states are mainly due to the $\mathrm{d}$ orbitals of $\mathrm{W}$ atoms for both heterostructures. However, we notice that there is a significant contribution from the $\mathrm{p}$ orbitals of the $\mathrm{B}$ atoms at $\mathrm{E}_{f}+2.8 \mathrm{eV}$ in the $\mathrm{WS}_{2} /$ $\mathrm{h}-\mathrm{BN}$ heterostructure. The states on the valance bands are generally due to hybridisation of the p orbitals of $\mathrm{S}$ and $\mathrm{N}$ atoms and $\mathrm{d}$ orbitals of $\mathrm{W}$ atoms in the $\mathrm{WS}_{2} / \mathrm{h}-\mathrm{BN}$ heterostructure. Furthermore, the contribution of $\mathrm{p}$ orbitals of $\mathrm{B}$ atoms in conduction band states is significantly reduced when $\mathrm{WS}_{2}$ was sandwiched into the h-BN bilayer. Similarly, the contribution of $\mathrm{p}$ orbitals of $\mathrm{N}$ atoms disappeared in valence band states resulting only in hybridisation between $\mathrm{p}$ orbitals on $\mathrm{S}$ atoms and d orbitals on $\mathrm{W}$ atoms. The PDOS of $\mathrm{WS}_{2} / \mathrm{MoS}_{2} / \mathrm{h}-\mathrm{BN}$ [see Figs. 11(a)-11(d)] shows that the VBM is mostly dominated by d orbitals of Mo and W atoms, while the CBM is mainly dominated by only d orbitals of Mo atoms. Moreover, there is a small contribution of $\mathrm{p}$ orbitals of $\mathrm{B}$ and $\mathrm{N}$ atoms to states in conduction bands and valence bands, respectively. The change in the PDOS when another h-BN monolayer is added on top of the $\mathrm{WS}_{2} / \mathrm{MoS}_{2} /$ $\mathrm{h}-\mathrm{BN}$ heterostructure is that the states in the VBM are due to the d orbitals of metal atoms and the p orbitals of $\mathrm{S}$ atoms in the $\mathrm{MoS}_{2}$ layer and those of $\mathrm{N}$ atoms, as shown in Fig. 11(d). The states in the CBM are due to the d orbitals of Mo atoms and $\mathrm{p}$ orbitals of $\mathrm{S}$ atoms in the $\mathrm{MoS}_{2}$ layer, while there is an absence of contribution of $\mathrm{p}$ orbitals of $\mathrm{S}$ atoms in the $\mathrm{WS}_{2}$ /
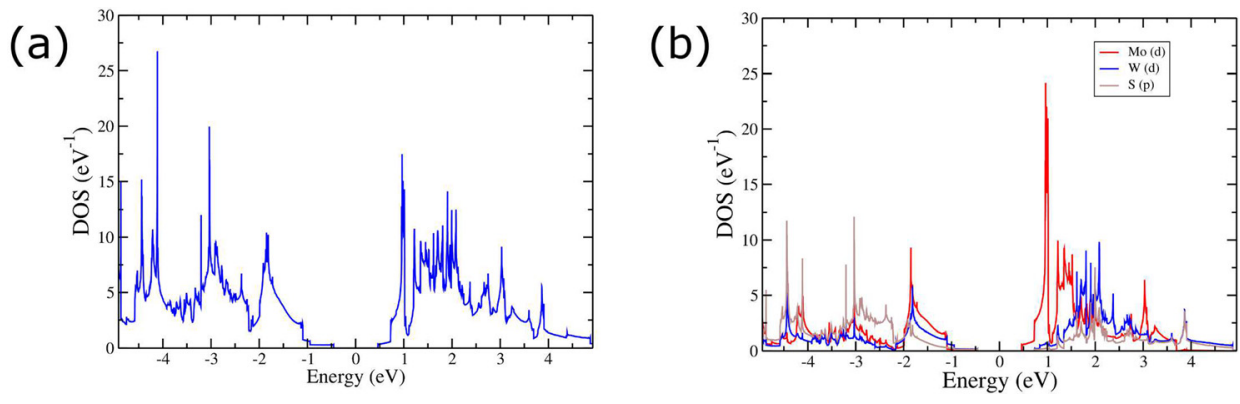

FIG. 8. Total density of states (TDOS) for (a) $\mathrm{WS}_{2} / \mathrm{MoS}_{2}$ and partial density of states (PDOS) for (b) $\mathrm{WS}_{2} / \mathrm{MoS}_{2}$ heterostructures. The Fermi level is at zero energy. 

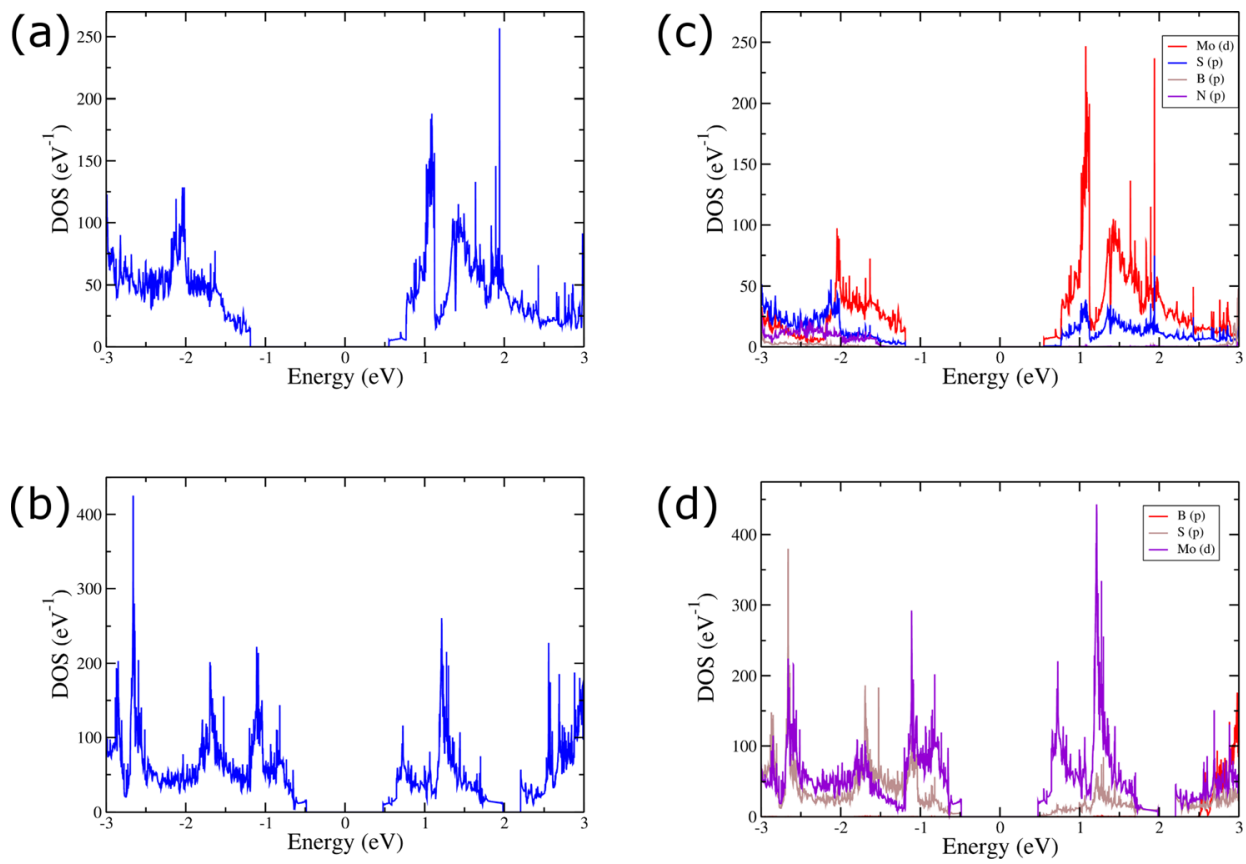

FIG. 9. Computed TDOS for (a) $\mathrm{MoS}_{2} / \mathrm{h}-\mathrm{BN}$ and (b) h-BN/MoS $2 / \mathrm{h}-\mathrm{BN}$ and PDOS for (c) $\mathrm{MoS}_{2} / \mathrm{h}-\mathrm{BN}$ and (d) $\mathrm{h}-\mathrm{BN} / \mathrm{MoS}_{2} / \mathrm{h}-\mathrm{BN}$. The Fermi level is at zero energy.

(a)

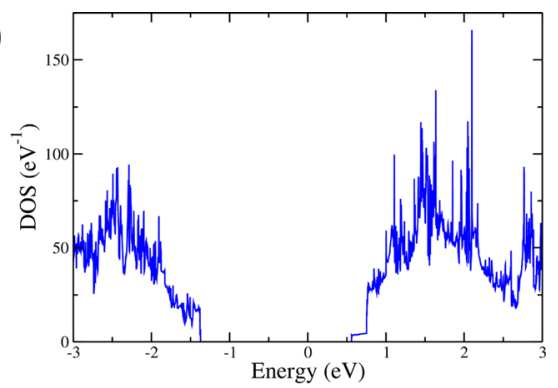

(b)

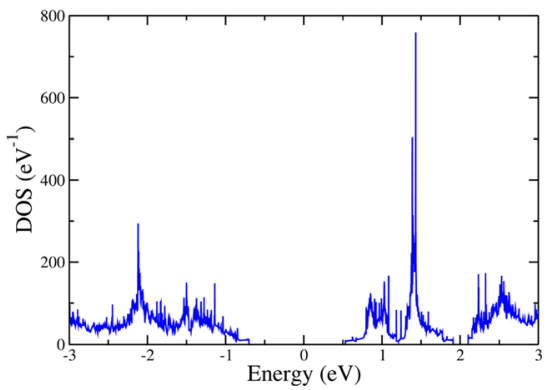

(c)

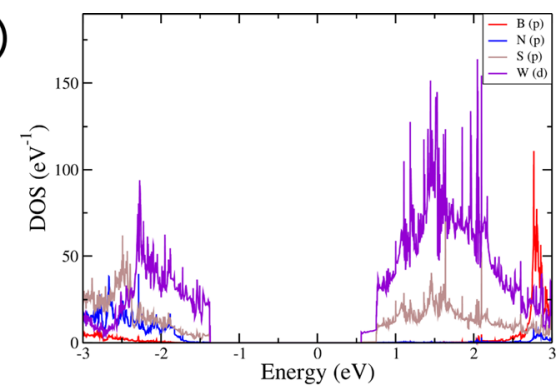

(d)

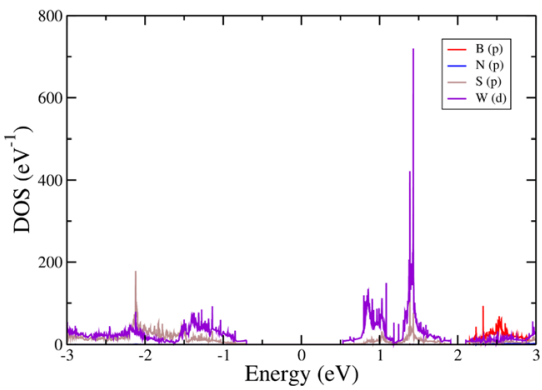

FIG. 10. Computed (TDOS) for (a) $\mathrm{WS}_{2} / \mathrm{h}-\mathrm{BN}$ and (b) h-BN/WS $2 / \mathrm{h}-\mathrm{BN}$ and PDOS for (c) $\mathrm{WS}_{2} / \mathrm{h}-\mathrm{BN}$ and (d) h-BN/WS $/$ h-BN heterostructures. The Fermi level is at zero energy.
$\mathrm{MoS}_{2} / \mathrm{h}$-BN heterostructure. We further notice that the contribution of $\mathrm{p}$ orbitals of $\mathrm{B}$ atoms in both top and bottom layers of $\mathrm{h}-\mathrm{BN}$ is significantly increased at $\mathrm{E}_{f}+3.150 \mathrm{eV}$ in the unoccupied region.

\section{CONCLUSION}

In conclusion, the electronic properties and dynamic stability of van der Waals heterostructures of h-BN/MoS $/$ h$\mathrm{BN}, \mathrm{h}-\mathrm{BN} / \mathrm{WS}_{2} / \mathrm{h}-\mathrm{BN}$, and $\mathrm{h}-\mathrm{BN} / \mathrm{WS}_{2} / \mathrm{MoS}_{2} / \mathrm{h}-\mathrm{BN}$ are investigated using density functional theory with a recently developed non-local van der Waals (vdW) density functional by Vydrov and Van Voorhis (rVV10). The calculated binding energies for different stacking orders confirm the dynamic stability of these heterostructures, and it was indicated that the van der Waals interaction between these two layers was not strong enough to make an abrupt structural change in TMDs. Therefore, the proposed heterostructures here offer a significant advantage to producing TMD based optoelectronic device applications. By analysing the electronic structures of the heterostructures, we found that all considered heterostructures are an indirect band gap semiconductor with the exception of the $\mathrm{WS}_{2} / \mathrm{h}-\mathrm{BN}$ heterostructure representing a direct band gap semiconductor. From band structure calculations, we conclude that the valence band maximum (VBM) at the $\Gamma$ point is very sensitive to interlayer interaction in the heterostructures which defines the location of the VBM at the $\Gamma$ or $\mathbf{K}$ point. Our PDOS calculations represent the negligible interaction between h-BN and TMDs. The states in the conduction band are also not affected by interlayer 
(a)

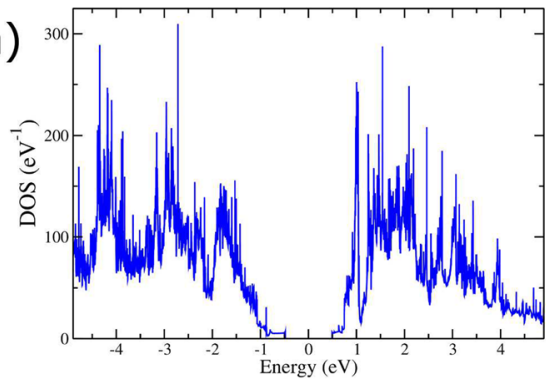

(b)

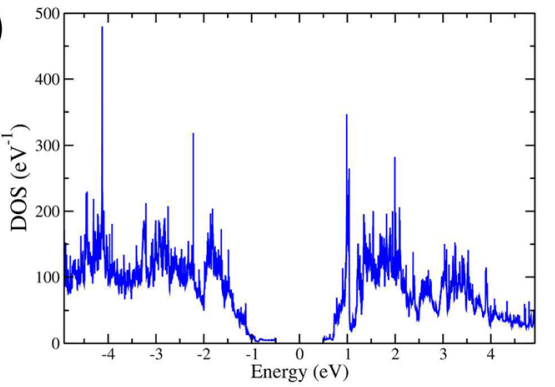

(c)

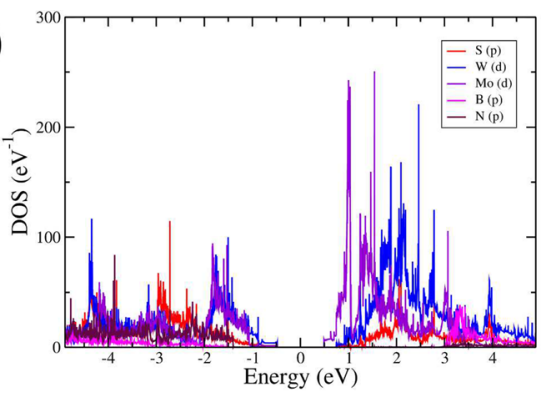

(d)

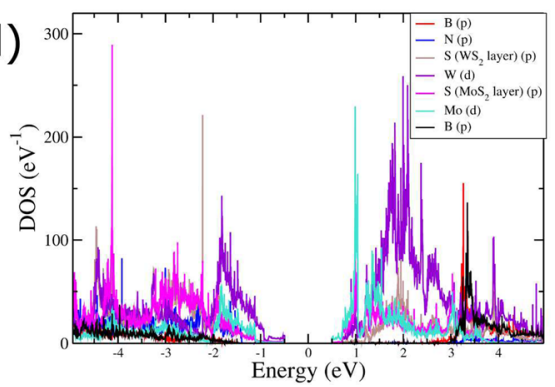

FIG. 11. Computed TDOS for (a) $\mathrm{WS}_{2} / \mathrm{MoS}_{2} / \mathrm{h}-\mathrm{BN}$ and (b) h-BN/WS $/$ $\mathrm{MoS}_{2} / \mathrm{h}-\mathrm{BN}$ and PDOS for (c) $\mathrm{WS}_{2} /$ $\mathrm{MoS}_{2} / \mathrm{h}-\mathrm{BN}$ and (d) h-BN/WS $/ \mathrm{MoS}_{2} /$ h-BN heterostructures. The Fermi level is at zero energy. interactions. This suggests that h-BN monolayer can be used to protect monolayer TMDs, and the heterostructures studied here offer significant advantages to producing TMD based optoelectronic device applications. We predict that the $\mathrm{WS}_{2} /$ h-BN heterostructure may be useful for solar energy conversion due to retaining its direct band gap. The direct band gap heterostructures should have higher efficiency in solar energy applications when compared with indirect band gap heterostructures. We believe that these results provide a support for experiments and serve as a guide for the development of novel 2D structures with high performance for photovoltaic and optoelectronic devices. More importantly, it is found that h-BN is an excellent candidate for the protection of intrinsic properties of $\mathrm{MoS}_{2}, \mathrm{WS}_{2}$, and $\mathrm{WS}_{2} / \mathrm{MoS}_{2}$ structures.

\section{ACKNOWLEDGMENTS}

OG acknowledges the support from the Scientific and Technological Research Council of Turkey (TUBITAK) under Project No. 115F024. The numerical calculations reported in this paper were fully performed at TUBITAK ULAKBIM, High Performance and Grid Computing Center (TRUBA resources).

${ }^{1}$ R. S. Sundaram, M. Engel, A. Lombardo, R. Krupke, A. C. Ferrari, P. Avouris, and M. Steiner, Nano Lett. 13, 1416 (2013).

${ }^{2}$ B. Radisavljevic and A. Kis, Nat. Mater. 12, 815 (2013).

${ }^{3}$ F. K. Perkins, A. L. Friedman, E. Cobas, P. M. Campbell, G. G. Jernigan, and B. T. Jonker, Nano Lett. 13, 668 (2013).

${ }^{4}$ Z. Y. Yin, H. Li, L. Jiang, Y. M. Shi, Y. H. Sun, G. Lu, Q. Zhang, X. D. Chen, and H. Zhang, ACS Nano 6, 74 (2012).

${ }^{5}$ M. Chhowalla, H. S. Shin, G. Eda, L. J. Li, K. P. Loh, and H. Zhang, Nat. Chem. 5, 263 (2013)

${ }^{6}$ H. Li, J. Wu, Z. Yin, and H. Zhang, Acc. Chem. Res. 47, 1067 (2014).

${ }^{7}$ X. Huang, C. Tan, Z. Yin, and H. Zhang, Adv. Mater. 26, 2185 (2014).

${ }^{8}$ D. Jariwala, V. K. Sangwan, L. J. Lauhon, T. J. Marks, and M. C. Hersam, ACS Nano 8, 1102 (2014).

${ }^{9}$ L. Britnell, R. M. Ribeiro, A. Eckmann, R. Jalil, B. D. Belle, A. Mishchenko, Y. J. Kim, R. V. Gorbachev, T. Georgiou, S. V. Morozov, A.
N. Grigorenko, A. K. Geim, C. Casiraghi, A. H. C. Neto, and K. S. Novoselov, Science 340, 1311 (2013).

${ }^{10}$ W. J. Yu, Y. Liu, H. Zhou, A. Yin, Z. Li, Y. Huang, and X. Duan, Nat. Nanotechnol. 8, 952 (2013).

${ }^{11}$ O. Lopez-Sanchez, D. Lembke, M. Kayci, A. Radenovic, and A. Kis, Nat. Nanotechnol. 8, 497 (2013).

${ }^{12}$ M. S. Choi, G. H. Lee, Y. J. Yu, D. Y. Lee, S. H. Lee, P. Kim, J. Hone, and W. J. Yoo, Nat. Commun. 4, 1624 (2013).

${ }^{13}$ R. Cheng, D. Li, H. Zhou, C. Wang, A. Yin, S. Jiang, Y. Liu, Y. Chen, Y. Huang, and X. Duan, Nano Lett. 10, 5590 (2014).

${ }^{14}$ J. Rohrer and P. Hyldgaard, Phys. Rev. B 83, 165423 (2011).

${ }^{15}$ R. Rao, R. Podila, R. Tsuchikawa, J. Katoch, D. Tishler, A. M. Rao, and M. Ishigami, ACS Nano 5, 1594 (2011).

${ }^{16}$ L. Britnell, R. V. Gorbachev, R. Jalil, B. D. Belle, F. Schedin, A. Mishchenko1, T. Georgiou1, M. I. Katsnelson, L. Eaves, S. V. Morozov, N. M. R. Peres, J. Leist, A. K. Geim, K. S. Novoselov, and L. A. Ponomarenko, Science 335, 947 (2012).

${ }^{17}$ G. H. Lee, Y. J. Yu, X. Cui, N. Petrone, C. H. Lee, M. S. Choi, D. Y. Lee, C. Lee, W. J. Yoo, K. Watanabe, T. Taniguchi, C. Nuckolls, P. Kim, and J. Hone, ACS Nano 7, 7931 (2013).

${ }^{18}$ S. Tongay, J. Zhou, C. Ataca, J. Liu, J. S. Kang, T. S. Matthews, L. You, J. B. Li, J. C. Grossman, and J. Q. Wu, Nano Lett. 13, 2831 (2013).

${ }^{19}$ H. Qiu, L. J. Pan, Z. N. Yao, J. J. Li, Y. Shi, and X. R. Wang, Appl. Phys. Lett. 100, 123104 (2012).

${ }^{20}$ H. J. Conley, B. Wang, J. I. Ziegler, R. F. Haglund, S. T. Pantelides, and K. I. Bolotin, Nano Lett. 13, 3626 (2013).

${ }^{21}$ A. Splendiani, L. Sun, Y. Zhang, T. Li, J. Kim, C. Y. Chim, G. Galli, and F. Wang, Nano Lett. 10, 1271 (2010).

${ }^{22}$ T. Korn, S. Heydrich, M. Hirmer, J. Schmutzler, and C. Schuller, Appl. Phys. Lett. 99, 102109 (2011).

${ }^{23}$ G. Eda, H. Yamaguchi, D. Voiry, T. Fujita, M. Chen, and M. Chhowalla, Nano Lett. 11, 5111 (2011).

${ }^{24}$ H. R. Gutierrez, N. Perea-Lopez, A. L. Elias, A. Berkdemyr, B. Wang, R. LV, F. Lopez-Urias, V. H. Crespi, H. Terrones, and M. Terrones, Nano Lett. 13, 3447 (2013).

${ }^{25}$ D. Xiao, G. B. Liu, W. X. Feng, X. D. Xu, and W. Yao, Phys. Rev. Lett. 108, 196802 (2012).

${ }^{26}$ H. L. Zeng, J. F. Dai, W. Yao, D. Xiao, and X. D. Cui, Nat. Nanotechnol. 7, 490 (2012)

${ }^{27}$ K. F. Mak, K. L. He, J. Shan, and T. F. Heinz, Nat. Nanotechnol. 7, 494 (2012).

${ }^{28}$ W. J. Yu, Z. Li, H. Zhou, Y. Chen, Y. Wang, Y. Huang, and X. Duan, Nat. Mater. 12, 246 (2013).

${ }^{29}$ Z. Liu, L. Song, S. Zhao, J. Huang, L. Ma, J. Zhang, J. Lou, and P. M. Ajayan, Nano Lett. 11, 2032 (2011). 
${ }^{30}$ Y. Shi, W. Zhou, A. Y. Lu, W. Fang, Y. H. Lee, A. L. Hsu, K. K. Kim, H. Y. Yang, L. J. Li, J. C. Idrobo, and J. Kong, Nano Lett. 12, 2784 (2012).

${ }^{31}$ G. W. Shim, K. Yoo, S. B. Seo, J. Shin, D. Y. Jung, I. S. Kang, G. W. Ahn, B. J. Cho, and S. Y. Choi, ACS Nano 8, 6655 (2014).

${ }^{32}$ X. Ling, Y. Lee, Y. Lin, W. Fang, L. Yu, M. S. Dresselhaus, and J. Kong, Nano Lett. 14, 464 (2014).

${ }^{33}$ M. Okada, T. Sawazaki, K. Watanabe, T. Taniguch, H. Hibino, H. Shinohara, and R. Kitaura, ACS Nano 8, 8273 (2014).

${ }^{34}$ X. Zhang, F. Meng, J. R. Christianson, J. C. Arroyo-Torres, M. A. Lukowski, D. Liang, J. R. Schmidt, and S. Jin, Nano Lett. 14, 3047 (2014).

${ }^{35}$ Y. Gong, J. Lin, X. Wang, G. Shi, S. Lei, Z. Lin, X. Zou, G. Ye, R. Vajtai, B. I. Yakobson, H. Terrones, M. Terrones, B. K. Tay, J. Lou, S. T. Pantelides, Z. Liu, W. Zhou, and P. M. Ajayan, Nat. Mater. 13, 1135 (2014).

${ }^{36}$ X. Cui, G. H. Lee, Y. D. Kim, G. Arefe, P. Y. Huang, C. H. Lee, D. A. Chenet, X. Zhang, L. Wang, F. Ye, F. Pizzocchero, B. S. Jessen, K. Watanabe, T. Taniguchi, D. A. Muller, T. Low, P. Kim, and J. Hone, Nat. Nanotechnol. 10, 534 (2015).

${ }^{37}$ A. M. Van der Zande, P. Y. Huang, D. A. Chenet, T. C. Berkelbach, Y. You, G. H. Lee, T. F. Heinz, D. R. Reichman, D. A. Muller, and J. C. Hone, Nat. Mater. 12, 554 (2013).

${ }^{38}$ S. Najmaei, Z. Liu, W. Zhou, X. Zou, G. Shi, S. Lei, B. I. Yakobson, J. C. Idrobo, P. M. Ajayan, and J. Lou, Nat. Mater. 12, 754 (2013).

${ }^{39}$ G. H. Han, N. J. Kybert, C. H. Naylor, B. S. Lee, J. Ping, J. H. Park, J. Kang, S. Y. Lee, Y. H. Lee, R. Agarwal, and A. C. Johnson, Nat. Commun. 6, 6128 (2015).

${ }^{40}$ Y. H. Lee, X. Q. Zhang, W. Zhang, M. T. Chang, C. T. Lin, K. D. Chang, Y. C. Yu, J. T. W. Wang, C. S. Chang, L. J. Li, and T. W. Lin, Adv. Mater. 24, 2320 (2012).

${ }^{41}$ R. Sabatini, T. Gorni, and S. de Gironcoli, Phys. Rev. B 87, 041108(R) (2013).
${ }^{42}$ P. Giannozzi, S. Baroni, N. Bonini, M. Calandra, R. Car, C. Cavazzoni, D. Ceresoli, G. L. Chiarotti, M. Cococcioni, I. Dabo, A. Dal Corso, S. Fabris, G. Gougoussis, A. Kokalj, M. Lazzeri, L. Martin-Samos, N. Marzari, F. Mauri, R. Mazzarello, S. Paolini, A. Pasquarello, L. Paulatto, C. Sbraccia, S. Scandolo, G. Sclauzero, A. P. Seitsonen, A. Smogunov, P. Umari, and R. M. Wentzcovitch, J. Phys.: Condens. Matter 21, 395502 (2009).

${ }^{43}$ T. Bjrkman, A. Gulans, A. V. Krasheninnikov, and R. M. Nieminen, Phys. Rev. Lett. 108, 235502 (2012).

${ }^{44}$ D. Vanderbilt, Phys. Rev. B 41, 7892 (1990).

${ }^{45}$ H. J. Monkhorst and J. D. Pack, Phys. Rev. B 13, 5188 (1976).

${ }^{46}$ M. Methfessel and A. T. Paxton, Phys. Rev. B 40, 3616 (1989).

${ }^{47}$ C. Ataca, H. Sahin, and S. Ciraci, J. Phys. Chem. C 116, 8983 (2012).

${ }^{48}$ H. L. Zhuang and R. G. Hennig, J. Phys. Chem. C 117, 20440 (2013).

${ }^{49}$ S. Lebegue and O. Eriksson, Phys. Rev. B 79, 115409 (2009).

${ }^{50}$ N. Lu, H. Guo, L. Li, J. Dai, L. Wang, W. N. Mei, X. Wu, and X. C. Zeng, Nanoscale 6, 2879 (2014).

${ }^{51}$ H. Zhang, X. B. Li, and L. M. Liu, J. Appl. Phys. 114, 093710 (2013).

${ }^{52}$ V. L. Solozhenko, G. Will, and F. Elf, Solid State Commun. 96, 1 (1995).

${ }^{53}$ H. Jiangang, K. Hummer, and C. Franchini, Phys. Rev. B 89, 075409 (2014).

${ }^{54}$ B. Amin, N. Singh, and U. Schwingenschlgl, Phys. Rev. B 92, 075439 (2015).

${ }^{55}$ Y. He, Y. Yang, Z. Zhang, Y. Gong, W. Zhou, Z. Hu, G. Ye, X. Zhang, E. Bianco, S. Lei, and Z. Jin, Nano Lett. 5, 3314 (2016).

${ }^{56}$ C. R. Dean, A. F. Young, I. Meric, C. Lee, L. Wang, S. Sorgenfrei, K. Watanabe, T. Taniguchi, P. Kim, K. L. Shepard, and J. Hone, Nat. Nanotechnol. 5, 722 (2010).

${ }^{57}$ K. F. Mak, C. Lee, J. Hone, J. Shan, and T. F. Heinz, Phys. Rev. Lett. 105, 136805 (2010).

${ }^{58}$ A. Kuc, N. Zibouche, and T. Heine, Phys. Rev. B 83, 245213 (2011). 